\title{
Prevalence and quantification of pathogenic Vibrio parahaemolyticus during shrimp culture in Thailand
}

\author{
Mingkwan Yingkajorn ${ }^{1,2}$, Pimonsri Mitraparp-arthorn ${ }^{1}$, Suphachai Nuanualsuwan $^{3}$, \\ Ratanaruji Poomwised ${ }^{1}$, Noochanat Kongchuay ${ }^{4}$, Natchaya Khamhaeng ${ }^{1}$, \\ Varaporn Vuddhakul ${ }^{1, *}$
}

\begin{abstract}
${ }^{1}$ Food Safety and Health Research Unit, Department of Microbiology, Faculty of Science, ${ }^{2}$ Department of Biomedical Science Faculty of Medicine, and ${ }^{4}$ Department of Mathematics, Faculty of Science, Prince of Songkla University, Hat Yai, Songkhla 90110, Thailand
\end{abstract}

${ }^{3}$ Faculty of Veterinary Science, Department of Veterinary Public Health, Chulalongkorn University, Bangkok 10330, Thailand

\begin{abstract}
Vibrio parahaemolyticus is a major cause of seafood-borne gastroenteritis. The human pathogenic strains possess $t d h$ or trh or both genes. In Thai shrimp farming, the level of pathogenic $V$. parahaemolyticus contamination has not been completely characterized, although it has been identified as a risk for people who consume undercooked shrimp. In this study, the prevalence and concentration of $V$. parahaemolyticus (total $\mathrm{Vp}$ ) and pathogenic $V$. parahaemolyticus $\left(t d h^{+} \mathrm{Vp}\right.$ and $t r h^{+} \mathrm{Vp}$ ) were investigated during shrimp culture cycles using the most probable number (MPN) method and were confirmed by PCR and the loop-mediated isothermal amplification (LAMP) techniques. The prevalence and concentration of total Vp were high in broodstock and egg samples at the start of the hatchery cycle, but the organism decreased in the subsequent larval and postlarval stages. In contrast, total Vp was low at the beginning of the pond cycle and dramatically increased during the later stages of culture. Broodstock and fresh feed were important sources of $V$. parahaemolyticus. Numbers of $t d h^{+} V p$ and $t r h^{+} V p$ detected by the LAMP technique were much greater than those detected by the PCR technique, especially in the late stages of the pond cycle. A direct correlation between total Vp and pathogenic Vp was demonstrated only during the harvest stage. This study will be useful as a guideline to establish levels of $V$. parahaemolyticus presence which can be considered as safe during shrimp culture. In addition, it could be used to identify the source of $V$. parahaemolyticus, which has recently been reported to be one of the etiologic agents of acute hepatopancreatic necrosis disease.
\end{abstract}

KEY WORDS: Vibrio parahaemolyticus $\cdot$ tdh gene $\cdot$ trh gene $\cdot$ Shrimp culture $\cdot$ LAMP Resale or republication not permitted without written consent of the publisher

\section{INTRODUCTION}

Vibrio parahaemolyticus is a Gram-negative halophilic bacterium found in marine environments worldwide. It is a leading cause of seafood-associated gastroenteritis. The most common symptoms in humans include watery diarrhea with abdominal cramps, nausea, vomiting and in some cases fever and headaches (Yeung \& Boor 2004). Not all strains of $V$. parahaemolyticus are pathogenic. V. parahaemolyticus strains that produce the major virulence factors, a thermostable direct hemolysin (TDH) or a TDH-related hemolysin (TRH), are considered to be human pathogenic strains (Takeda 1982, Honda et. al. 1987). TDH and TRH are encoded by the $t d h$ and trh genes, respectively. The number of $V$. parahaemolyticus isolates that possess $t d h$ or trh genes in the environment varies, and the number determined 
depends on the location and detection techniques. However, around $90 \%$ of clinical isolates possess $t d h$, trh, or both genes (Deepanjali et al. 2005, Wootipoom et al. 2007, Gutierrez West et al. 2013). Most infections due to $V$. parahaemolyticus have been associated with shellfish consumption. In Hong Kong, around $40 \%$ of $V$. parahaemolyticus associated with food poisoning is caused by consumption of shrimp (Liu \& Chen 2013). Investigation of 28 isolates of $V$. parahaemolyticus obtained from shrimp isolated from the south coast of Iran revealed that $5(1.7 \%)$ and $2(0.7 \%)$ isolates were $t d h^{+}$and $t r h^{+}$, respectively (Rahimi et al. 2010). In Malaysia, testing of $128 \mathrm{~V}$. parahaemolyticus isolates from frozen and cultured shrimp, including some from the culture environment, revealed that $14(10.9 \%)$ and $4(3.1 \%)$ were positive for the $t d h$ and trh genes, respectively, and this indicated that healthy people are at risk after consumption of raw or undercooked shrimp (Sujeewa et al. 2009).

Thailand is one of the world's leading exporters of shrimp. In shrimp farming, especially in Thailand, the level of $t d h^{+}$or $t r h^{+} V$. parahaemolyticus, as well as the source of this bacterium which is present during each shrimp cultivation cycle, is still limited, although the potential for infection of consumers cannot be ruled out. The aims of this study were to investigate the prevalence and concentration of potential infectious $V$. parahaemolyticus in shrimp cultured from the hatchery to the harvest cycle and to evaluate the probability of detecting pathogenic $V$. parahaemolyticus during each of the stages of shrimp culture. In addition, we also applied the loop-mediated isothermal amplification (LAMP) technique to detect pathogenic $V$. parahaemolyticus in shrimp culture.

\section{MATERIALS AND METHODS}

\section{Scenario study}

In this study, the prevalence and concentration of Vibrio parahaemolyticus was evaluated by following the shrimp culturing cycle from the hatchery, to the pond, to harvest. Feed was also investigated because this factor was involved in most stages of the cultivation processes. The study was based on a multiple stage scenario from broodstock, shrimp spawn (egg), larva hatch (nauplius, protozoea and mysis), postlarva (PL) development, and juvenile (1.5 mo culture) to adult (3-4.5 mo culture) development. At each stage, the prevalence and concentration of V. parahaemolyticus were determined. Parameters used in this study were the presence or absence of $V$. parahaemolyticus (hereafter termed total $\mathrm{Vp}$ ) and the presence or absence of human pathogenic $t d h^{+}$or $t r h^{+} V$. parahaemolyticus (hereafter termed $t d h^{+} \mathrm{Vp}$ and $t r h^{+} \mathrm{Vp}$, respectively). Quantitative evaluation was expressed as a geometric mean of the most probable number (MPN) of $V$. parahaemolyticus per $\mathrm{g}$ or $\mathrm{ml}\left(\mathrm{MPN} \mathrm{g}^{-1}\right.$ or $\mathrm{ml}^{-1}$ ) of sample. When SD $<0.01 \log _{10} \mathrm{MPN}$, it is reported as zero (0). The probability of detection of total Vp and pathogenic $V$. parahaemolyticus as well as the correlation between total $\mathrm{Vp}, t d h^{+} \mathrm{Vp}$, and $t r h^{+} \mathrm{Vp}$ were also determined (see the Appendix).

\section{Sample collection}

Samples were collected from 2 different shrimp farms (Litopenaeus vanamei shrimp) and 2 hatcheries located in the Ranode district, Songkhla Province, Thailand, between June 2010 and February 2012. After collection, they were kept in sterile containers and brought to the laboratory as quickly as possible. The prevalence and concentration of $V$. parahaemolyticus were determined using the MPN technique for the hatchery, pond, and harvest cycles including feed. Various quantities of samples were obtained $(3,10$, or $25 \mathrm{ml}$ or $25 \mathrm{~g})$ depending on the type of sample (Table 1). In the hatchery cycle, the broodstock $(25 \mathrm{~g})$, water $(25 \mathrm{ml})$ and eggs $(3 \mathrm{ml}$ containing more than 30000 eggs) were collected from each farm (Table 1). In addition, $3 \mathrm{ml}$ (more than 25000 larva) containing each stage of the larva (nauplii, protozoea, and mysis) was examined. For the PL stage, PL Day $1\left(\mathrm{PL}_{1}\right)$ and PL Day 11-15 ( $\left.\mathrm{PL}_{11-15}\right)$, $25 \mathrm{ml}$ (containing 25-30 PL) were tested. In the pond cycle, stages of shrimp culture can be categorized as follows: PA (pond preparation), PB (water filling), PC (water treatment), PD (postlarva release), PE1 (1.5 mo culture) and PE2 (3.0 mo culture). In this cycle as well as the harvest cycle (PE3: 4.5 mo culture), water (25 $\mathrm{ml}$ from 5 different locations), and sediment $(25 \mathrm{~g}$ from 3 different locations in the pond) were collected for each stage (Table 1). In addition, for PE1 to PE3, shrimp (25 g) were obtained for $V$. parahaemolyticus investigations. For the feed at least $10 \mathrm{ml}$ or $25 \mathrm{~g}$ of natural feed, fresh feed, or formulated feed from 2 different companies were investigated.

\section{Bacterial enumeration and confirmation}

Alkaline peptone water (APW) was used as the enrichment medium for the culture of $V$. parahaemo- 
Table 1. Types and quantity of sample collected at each stage of shrimp culture. $\mathrm{PL}_{1}$ : postlarva Day $1 ; \mathrm{PL}_{11-15}$ : postlarva Day 11-15; PA: pond preparation stage; $\mathrm{PB}$ : water filling stage; $\mathrm{PC}$ : water treatment stage PD: postlarva release stage; PE1: 1.5 mo culturing stage; PE2: 3 mo culturing stage; PE3: 4.5 mo culturing (harvest) stage

\begin{tabular}{|c|c|c|c|c|}
\hline Cycle & Stage(s) & $\begin{array}{l}\text { Sample } \\
\text { type }\end{array}$ & $\begin{array}{l}\text { No. of samples } \\
\text { collected }\end{array}$ & $\begin{array}{l}\text { Quantity } \\
\text { of sample }\end{array}$ \\
\hline \multirow[t]{8}{*}{ Hatchery } & \multirow[t]{2}{*}{ Broodstock } & Broodstock & 8 & $25 \mathrm{~g}$ \\
\hline & & Water & 20 & $25 \mathrm{ml}$ \\
\hline & Egg & Egg & 4 & $3 \mathrm{ml}$ \\
\hline & Nauplii & Nauplii & 4 & $3 \mathrm{ml}$ \\
\hline & Protozoea & Protozoea & 4 & $3 \mathrm{ml}$ \\
\hline & Mysis & Mysis & 4 & $3 \mathrm{ml}$ \\
\hline & $\mathrm{PL}_{1}$ & $\mathrm{PL}_{1}$ & 4 & $3 \mathrm{ml}$ \\
\hline & $\mathrm{PL}_{11-15}$ & $\mathrm{PL}_{11-15}$ & 4 & $3 \mathrm{ml}$ \\
\hline \multirow[t]{15}{*}{ Pond } & \multirow[t]{2}{*}{ PA } & Sediment & 24 & $25 \mathrm{~g}$ \\
\hline & & Water & 24 & $25 \mathrm{ml}$ \\
\hline & \multirow[t]{2}{*}{ PB } & Sediment & 24 & $25 \mathrm{~g}$ \\
\hline & & Water & 47 & $25 \mathrm{ml}$ \\
\hline & \multirow[t]{2}{*}{$\mathrm{PC}$} & Sediment & 24 & $25 \mathrm{~g}$ \\
\hline & & Water & 40 & $25 \mathrm{ml}$ \\
\hline & \multirow[t]{3}{*}{$\mathrm{PD}$} & PL & 24 & $3 \mathrm{ml}$ \\
\hline & & Sediment & 24 & $25 \mathrm{~g}$ \\
\hline & & Water & 40 & $25 \mathrm{ml}$ \\
\hline & \multirow[t]{3}{*}{ PE1 } & Shrimp & 24 & $25 \mathrm{~g}$ \\
\hline & & Sediment & 24 & $25 \mathrm{~g}$ \\
\hline & & Water & 40 & $25 \mathrm{ml}$ \\
\hline & \multirow[t]{3}{*}{ PE2 } & Shrimp & 24 & $25 \mathrm{~g}$ \\
\hline & & Sediment & 24 & $25 \mathrm{~g}$ \\
\hline & & Water & 40 & $25 \mathrm{ml}$ \\
\hline \multirow[t]{3}{*}{ Harvest } & \multirow[t]{3}{*}{ PE3 } & Shrimp & 24 & $25 \mathrm{~g}$ \\
\hline & & Sediment & 24 & $25 \mathrm{~g}$ \\
\hline & & Water & 40 & $25 \mathrm{ml}$ \\
\hline \multirow[t]{3}{*}{ Feed } & & Natural feed ${ }^{a}$ & 28 & $10 \mathrm{ml}$ \\
\hline & & Fresh feed $^{\mathrm{b}}$ & 18 & $25 \mathrm{~g}$ \\
\hline & & Formulated feed & 32 & $25 \mathrm{~g}$ \\
\hline
\end{tabular}

lyticus. Each sample was homogenized with APW, with a $\mathrm{pH}$ of 8.6 , at a ratio of $1: 10$, and the supernatant was transferred for inoculation into 3 tubes to be investigated using the MPN procedure. Briefly, $10,1,0.1,0.01$, or $0.001 \mathrm{ml}$ of the supernatant were inoculated into APW in triplicate. Inoculation of the 0.01 and $0.001 \mathrm{ml}$ samples was performed by transferring $1 \mathrm{ml}$ of the 0.1 and 0.01 inocula, respectively, in $9 \mathrm{ml}$ of APW. For the egg, larva, and PL stages, $1 \mathrm{ml}$ of the obtained sample was transferred to the first set of MPN dilution tubes (3 tubes in each set; total amount transferred $3 \mathrm{ml}$ ). Inoculated tubes were incubated for $18 \mathrm{~h}$ at $37^{\circ} \mathrm{C}$. The presence or absence of $V$. parahaemolyticus (total $\mathrm{Vp}$ ), $t d h^{+} \mathrm{Vp}$, and $t r h^{+}$ $\mathrm{Vp}$ in each tube of the enrichment culture was examined using the colony isolation-PCR method (defined as colony PCR). Briefly, 1 loopful of APW was taken from each of the tubes showing turbidity and was streaked onto CHROMagar Vibrio (CHROMagar Microbiology). After overnight incubation at $37^{\circ} \mathrm{C}$, up to 5-10 mauve colonies most likely to be $V$. parahaemolyticus were selected from each CHROMagar Vibrio plate. Each of the selected colonies was inoculated into Luria-Bertani (LB) broth medium containing $1 \% \mathrm{NaCl}$ and incubated at $37^{\circ} \mathrm{C}$ with shaking (160 rpm) overnight. One $\mathrm{ml}$ of the overnight culture was examined for the $V$. parahaemolyticus tox $R$, tdh and trh genes using the PCR technique (colony PCR) (Yamamoto et al. 2008). The presence or absence of total $\mathrm{Vp}, t d h^{+} \mathrm{Vp}$, and $t r h^{+} \mathrm{Vp}$ in each tube was determined based on the detection of these 3 genes, respectively, in the selected colonies. If at least 1 colony was PCR positive, the corresponding tube and sample were considered positive for $V$. parahaemolyticus and used to calculate the MPN for the concentration of total $\mathrm{Vp}, t d h^{+} \mathrm{Vp}$, and $t r h^{+} \mathrm{Vp} \mathrm{g}^{-1}\left(\right.$ or $\mathrm{ml}^{-1}$ ) in the corresponding sample (M. Curiale, http://i2workout.com/mcuriale/mpn/index. html). However, the 5-10 mauve colonies selected by CHROMagar Vibrio using colony PCR may not represent the whole population of $V$. parahaemolyticus in the sample. Therefore, the supernatant of all turbid MPN tubes was confirmed for $t d h^{+} \mathrm{Vp}$ and $t r h^{+} \mathrm{Vp}$ using the LAMP assay, and the numbers in the positive tube were used to calculate the MPN as described above.

\section{LAMP assay}

One $\mathrm{ml}$ of each positive tube was centrifuged at $900 \times g$ for $1 \mathrm{~min}$ to remove large debris. The supernatant was collected and centrifuged at $10000 \times g$ for $5 \mathrm{~min}$. This pellet was resuspended in $500 \mu \mathrm{l}$ sterile distilled water, boiled for $10 \mathrm{~min}$, then immediately cooled and centrifuged at $20000 \times g$ at $4^{\circ} \mathrm{C}$ for $5 \mathrm{~min}$. The clear supernatant was used as the DNA template for LAMP detection of the $t d h$ and trh genes (Yamazaki et al. 2010). Briefly, $25 \mu$ of LAMP reaction mixture contained $1.6 \mu \mathrm{M}$ each of the inner primers FIP and BIP, $0.2 \mu \mathrm{M}$ each of the outer primers F3 and B3, $0.8 \mu \mathrm{M}$ each of the loop primers LF and LB, $12.5 \mu$ l of $2 \times$ LAMP reaction buffer, $1.0 \mu$ of $B s t$ polymerase, and $5 \mu \mathrm{l}$ of template DNA. After being gently mixed, it was then incubated at $65^{\circ} \mathrm{C}$ for 
60 min and inactivated at $80^{\circ} \mathrm{C}$ for 2 min to complete the reaction. DNA amplification was monitored with a real-time turbidimeter (RT-160C, Eiken Chemical). A reaction was considered positive when the turbidity reached 0.1 within $60 \mathrm{~min}$ at $650 \mathrm{~nm}$ for the $t d h$ LAMP and within $90 \mathrm{~min}$ at $650 \mathrm{~nm}$ for the trh-LAMP. The presence of a white precipitation visible to the naked eye was considered a positive result. In addition, the presence of $V$. parahaemolyticus in all positive tubes was confirmed using tlh as a target gene (Yamazaki et al. 2008). The sensitivity and specificity of the LAMP technique for detection of pathogenic $V$. parahaemolyticus were calculated as in a previous study (Lau et al. 2010) and compared to the PCR method. Sensitivity was defined as (number of samples positive for both techniques)/(number of samples positive for both techniques + number of samples positive for PCR but negative for LAMP), and specificity was defined as (number of samples negative for both techniques)/(number of samples negative for both techniques + number of samples positive for LAMP but negative for PCR). This approach assumes that PCR is a gold standard test.

\section{RESULTS}

\section{Prevalence and concentration of total Vp}

For the hatchery cycle, the prevalence of Vibrio parahaemolyticus in both the male and female broodstocks, including water in the stocking tank, was investigated in a total of 28 samples. All of them $(100 \%)$ were positive for total $\mathrm{Vp}$ (Table 2). In addition, all the egg and nauplii samples were positive for total $\mathrm{Vp}$; however, only $25-50 \%$ of the samples obtained from the protozoea, mysis, $\mathrm{PL}_{1}$ and $\mathrm{PL}_{11-15}$ were positive. In the pond cycle, at the PA-PD stages, $15.9-43.8 \%$ of samples were positive for total $\mathrm{Vp}$, and the number of positive samples increased to $94.3 \%$ after the shrimp had been cultured for 1.5 mo (PE1) (Table 2). It was of interest that after culture for 3 mo (PE2) and 4.5 mo (PE3) (harvest cycle) all samples were positive for total Vp.
The concentration of total $\mathrm{Vp}$ in the broodstock, egg, larva, PL and shrimp was evaluated for each stage of the shrimp culture. The mean and standard deviation of the concentration of total Vp detected in the hatchery cycle was high in the broodstock and egg samples $\left(1.93 \pm 0.02\right.$ and $1.99 \pm 0 \log _{10} \mathrm{MPN} \mathrm{g}^{-1}$ or $\mathrm{ml}^{-1}$, respectively) (Fig. 1), and it then decreased significantly in the larval and PL stages $(-0.18 \pm 0$ to $-0.53 \pm 0 \log _{10} \mathrm{MPN} \mathrm{g}{ }^{-1}$ or $\mathrm{ml}^{-1}$ ). However, after release of the PL into the pond at the PD stage, the number of total $\mathrm{Vp}$ continuously increased during the PE1 to PE3 stages (1.62 \pm 0 to $2.11 \pm 0 \log _{10}$ MPN $\mathrm{g}^{-1}$ or $\mathrm{ml}^{-1}$ ).

In the pond cycle, the average concentrations of total $\mathrm{Vp}$ in the sediment and water samples in the PA stage were $0.46 \pm 0.02$ and $-0.21 \pm 0 \log _{10} \mathrm{MPN} \mathrm{g}^{-1}$ or $\mathrm{ml}^{-1}$, respectively, and they then decreased in the PB to PD stages (Fig. 2). After that the total Vp continuously increased during the PE1 to PE3 stages.

Investigation of $V$. parahaemolyticus in the shrimp feed showed that 3.6 and $83.3 \%$ of total Vp were
Table 2. Prevalence of total Vibrio parahaemolyticus (total Vp) and pathogenic $V$. parahaemolyticus $\left(t d h^{+} \mathrm{Vp}\right.$ and $t r h^{+} \mathrm{Vp}$ ) in shrimp culture as determined by PCR or loop-mediated isothermal amplification (LAMP), presented as 'no. of positive samples total'. $\mathrm{PL}_{1}$ : postlarva Day 1; postlarva Day 11-15; PA: pond preparation stage; $\mathrm{PB}$ : water filling stage; $\mathrm{PC}$ : water treatment stage; $\mathrm{PD}$ : postlarva release stage; PE1: 1.5 mo culturing stage; PE2: 3 mo culturing stage; PE3: 4.5 mo culturing (harvest) stage. MPN: most probable number; ND: not determined

\begin{tabular}{|c|c|c|c|c|c|}
\hline \multirow{2}{*}{$\begin{array}{l}\text { Shrimp } \\
\text { culture } \\
\text { stage(s) }\end{array}$} & \multirow{2}{*}{$\begin{array}{c}\text { Total Vp } \\
(\%)\end{array}$} & \multicolumn{2}{|c|}{$-\mathrm{PCR}-$} & \multicolumn{2}{|c|}{$\longrightarrow$ LAMP } \\
\hline & & $\begin{array}{c}t d h^{+} \mathrm{Vp} \\
(\%)\end{array}$ & $\begin{array}{c}t r h^{+} V p \\
(\%)\end{array}$ & $\begin{array}{c}t d h^{+} V p \\
(\%)\end{array}$ & $\begin{array}{c}t r h^{+} V p \\
(\%)\end{array}$ \\
\hline \multicolumn{6}{|l|}{ Hatchery } \\
\hline Broodstock & $28 / 28(100)$ & $5 / 28(17.8)$ & $1 / 28(3.6)$ & 5/28 (17.8) & $2 / 28(7.1)$ \\
\hline Egg & $4 / 4(100)$ & $0^{\mathrm{a}} / 4$ & $0 / 4$ & $0 / 4$ & $0 / 4$ \\
\hline Nauplii & $4 / 4(100)$ & $0 / 4$ & $0 / 4$ & $0 / 4$ & $0 / 4$ \\
\hline Protozoea & $1 / 4(25)$ & $0 / 1$ & $0 / 1$ & $0 / 1$ & $0 / 1$ \\
\hline Mysis & $1 / 4(25)$ & $0 / 1$ & $0 / 1$ & $0 / 1$ & $0 / 1$ \\
\hline $\mathrm{PL}_{1}$ & $1 / 4(25)$ & $0 / 1$ & $0 / 1$ & $0 / 1$ & $0 / 1$ \\
\hline$P_{11-15}$ & $2 / 4(50)$ & $0 / 2$ & $0 / 2$ & $0 / 2$ & $0 / 2$ \\
\hline \multicolumn{6}{|l|}{ Pond } \\
\hline PA & $21 / 48(43.8)$ & $0 / 21$ & $0 / 21$ & $0 / 21$ & $0 / 21$ \\
\hline PB & $12 / 71(16.9)$ & $0 / 12$ & $0 / 12$ & $0 / 12$ & $0 / 12$ \\
\hline PC & $14 / 64(21.9)$ & $0 / 14$ & $0 / 14$ & $0 / 14$ & $0 / 14$ \\
\hline PD & $14 / 88(15.9)$ & $0 / 14$ & $0 / 14$ & $0 / 14$ & $0 / 14$ \\
\hline PE1 & 83/88 (94.3) & $0 / 83$ & $0 / 83$ & 1/83 (1.2) & $0 / 83$ \\
\hline PE2 & 88/88 (100) & $0 / 88$ & $0 / 88$ & $10 / 88(11.4)$ & $0 / 88$ \\
\hline \multicolumn{6}{|l|}{ Harvest } \\
\hline PE3 & 88/88 (100) & $0 / 88$ & $0 / 88$ & $14 / 88(15.9)$ & $4 / 88(4.6)$ \\
\hline \multicolumn{6}{|l|}{ Feed } \\
\hline Natural & $1 / 28(3.6)$ & $0 / 1$ & $0 / 1$ & $0 / 1$ & $0 / 1$ \\
\hline Fresh & 15/18 (83.3) & 4/15 (26.7) & $0 / 15$ & 8/15 (53.3) & $3 / 15$ (20) \\
\hline Formulated & $0 / 32$ & ND & ND & ND & ND \\
\hline${ }^{\mathrm{a}}<0.3 \mathrm{MPN}$ & or $\mathrm{ml}^{-1}$ & & & & \\
\hline
\end{tabular}




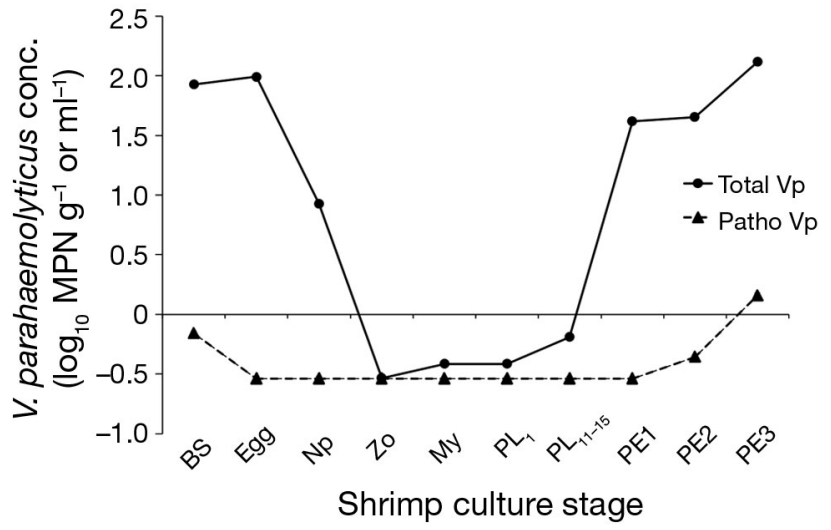

Fig. 1. Mean concentration of total Vibrio parahaemolyticus (total $\mathrm{Vp}$ ) and pathogenic $V$. parahaemolyticus (patho $\mathrm{Vp}$ ) in shrimp culture stages. BS: broodstock; Np: nauplii; Zo: protozoea; My: mysis; $\mathrm{PL}_{1}$ : postlarva Day 1; $\mathrm{PL}_{11-15}$ : postlarva Day 11-15; PE1: 1.5 mo culturing stage; PE2: 3 mo culturing stage; PE3: 4.5 mo culturing (harvest) stage. MPN: most probable number

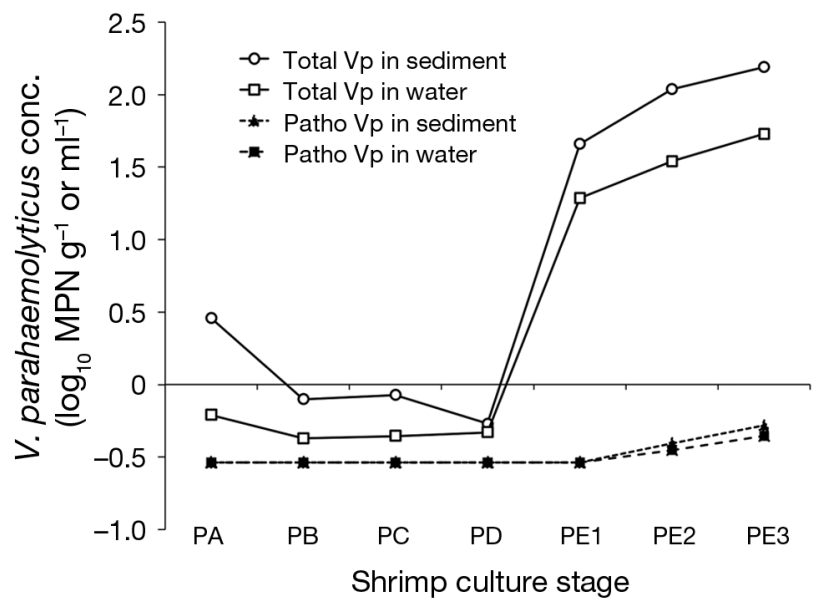

Fig. 2. Mean concentration of total Vibrio parahaemolyticus (total $\mathrm{Vp}$ ) and pathogenic $V$. parahaemolyticus (patho $\mathrm{Vp}$ ) in the pond sediment and water. PA: pond preparation; PB: water filling; PC: water treatment; PD: postlarva release; PE1: 1.5 mo culturing stage; PE2: 3 mo culturing stage; PE3: 4.5 mo culturing (harvest) stage. MPN: most probable number

detected in the natural feed and fresh feed, respectively (Table 2). Natural feed contained artemia and spirulina whereas fresh feed was composed of bloodworm, sandworm, squid, and shell. Thus, it is not surprising that $V$. parahaemolyticus was detected mostly in the fresh feed. The average concentrations of the total $\mathrm{Vp}$ detected were between $-0.47 \pm 0.40$ and $1.45 \pm 0.04 \log _{10} \mathrm{MPN} \mathrm{g}^{-1}$ or ml ${ }^{-1}$ (Fig. 3). No V. parahaemolyticus was observed in the formulating feed $\left(-0.53 \pm 0 \log _{10} \mathrm{MPN} \mathrm{g}^{-1}\right)$.

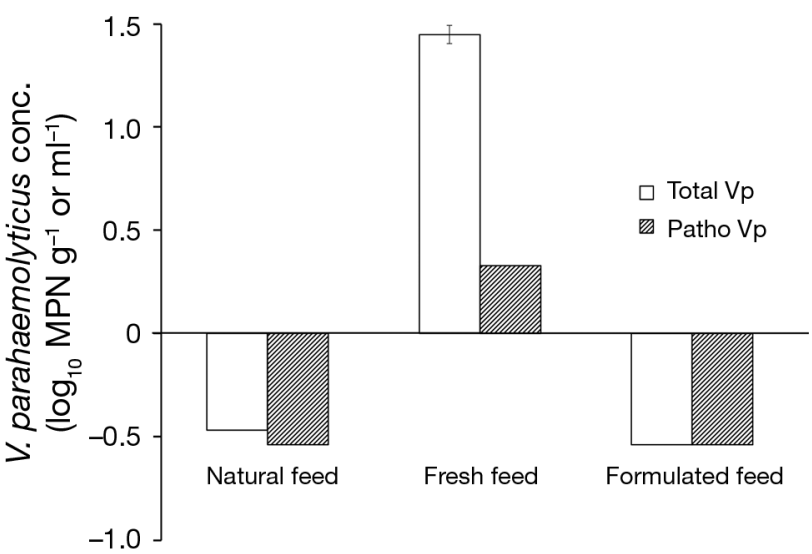

Fig. 3. Mean concentration of total Vibrio parahaemolyticus (total $\mathrm{Vp}$ ) and pathogenic V. parahaemolyticus (pathoVp) in feed. Natural feed: artemia and spirulina; fresh feed: bloodworm, sandworm, squid, and shell. MPN: most probable number

\section{Prevalence and concentration of $t d h^{+} \mathrm{Vp}$}

Using the PCR technique for detection of pathogenic $V$. parahaemolyticus in broodstock, 5 samples and 1 sample were positive for $t d h^{+} \mathrm{Vp}$ and $t r h^{+} \mathrm{Vp}$, respectively, whereas using the LAMP technique, an additional $t \mathrm{rh}^{+} \mathrm{Vp}$ was detected (Table 2). No pathogenic $V$. parahaemolyticus was detected in the egg samples or in any of the larval stages by either the PCR or the LAMP technique. At the PA-PD stages, all samples were negative for $t d h^{+} \mathrm{Vp}$ and $t r h^{+} \mathrm{Vp}$, but these were detected during the PE1-PE3 stages by the LAMP technique (Table 2). The prevalence of $t d h^{+} \mathrm{Vp}$ increased from 1.2 to 11.4 and $15.9 \%$ for the PE1, PE2 and PE3 stages, respectively. Around 4.6\% of $\mathrm{rh}^{+} \mathrm{Vp}$ was detected in the harvest stage. From the fresh feed, $26.7 \%$ of samples were positive for $t d h^{+} \mathrm{Vp}$ as determined by the PCR technique whereas, using the LAMP technique, the number of positive samples increased to $53.3 \%$. In addition, $20.0 \%$ of samples were found to be positive for $t r h^{+} \mathrm{Vp}$ using this technique.

In order to compare the sensitivity and specificity of PCR to the LAMP technique for detection of pathogenic $V$. parahaemolyticus, a total of 377 positive samples of $V$. parahaemolyticus were investigated (Table 2). PCR detected $t d h^{+} \mathrm{Vp}$ and $t r h^{+} \mathrm{Vp}$ in 9 and 1 samples, respectively, whereas LAMP detected $t d h^{+} \mathrm{Vp}$ and $t r h^{+} \mathrm{Vp}$ in 38 and 9 samples, respectively (Table 3). In addition, 339 and 368 samples were negative for the $t d h$ and trh genes, respectively, by both LAMP and PCR. Thus, using PCR as the standard method for detection of virulent genes of $V$. para- 
Table 3. Sensitivity and specificity of loop-mediated isothermal amplification (LAMP) compared to PCR for detection of pathogenic Vibrio parahaemolyticus

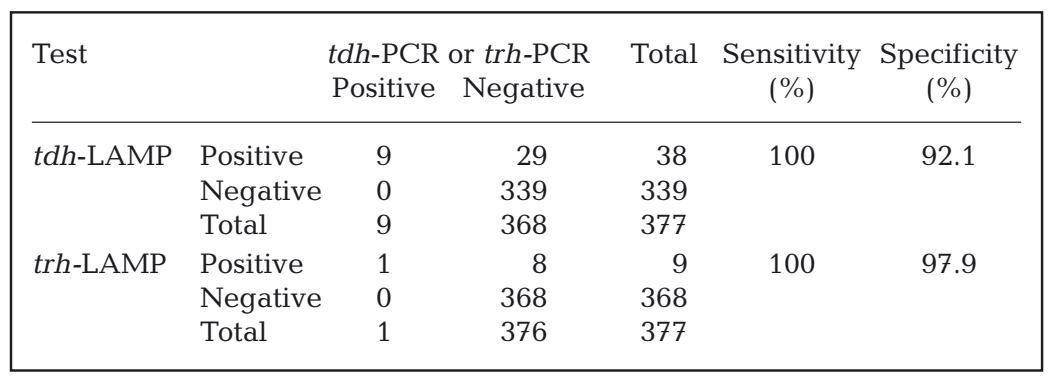

haemolyticus, sensitivity and specificity of $t d h$-LAMP were 100 and $92.1 \%$, respectively, whereas sensitivity and specificity of trh-LAMP were 100 and $97.9 \%$, respectively (Table 3 ).

The average concentrations of pathogenic V. parahaemolyticus detected in broodstock, eggs, larva, PL, and shrimp were investigated. The highest concentration $\left(0.16 \pm 0.03 \log _{10}\right.$ MPN g $\left.{ }^{-1}\right)$ was detected in shrimp at the PE3 stage (Fig. 1). In the pond cycle, the concentrations of pathogenic $V$. parahaemolyticus in the sediment and water increased simultaneously during the PE2 and PE3 stages (Fig. 2). In the feed, pathogenic $V$. parahaemolyticus was detected only in the fresh feed with an average concentration of $0.30 \pm 0 \log _{10} \mathrm{MPN} \mathrm{g}^{-1}$ (Fig. 3).

The probability of detection of total Vp or pathogenic $V$. parahaemolyticus in shrimp from at least one source of contamination fluctuated from the broodstock to the harvest stage (Fig. 4). This finding

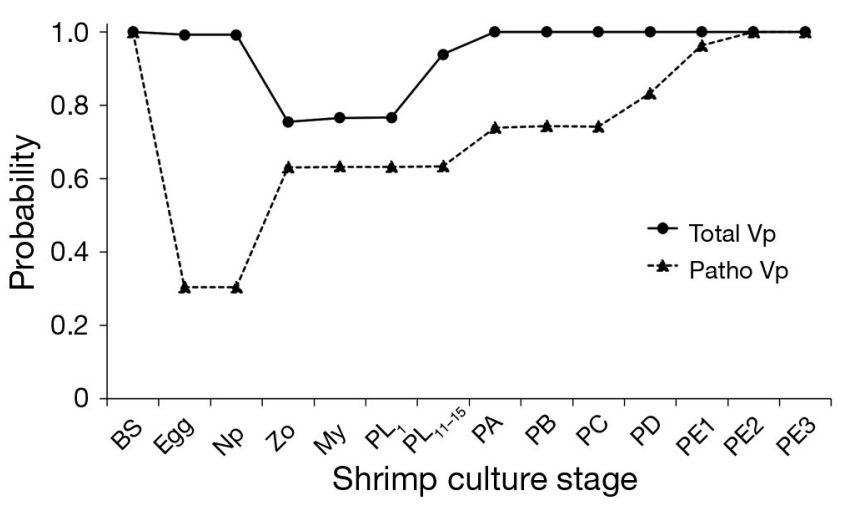

Fig. 4. Probability of detection of total Vibrio parahaemolyticus (total $\mathrm{Vp}$ ) and pathogenic V. parahaemolyticus (patho $\mathrm{Vp}$ ) in shrimp culture. BS: broodstock; Np: nauplii; Zo: protozoea; My: mysis; PA: pond preparation; PB: water filling; PC: water treatment; $\mathrm{PD}$ : postlarva release; $\mathrm{PL}_{1}$ : postlarva Day 1; $\mathrm{PL}_{11-15}$ : postlarva Day 11-15; PE1: 1.5 mo culturing stage; PE2: 3 mo culturing stage; PE3: 4.5 mo culturing (harvest) stage was compatible with the prevalence and concentration of $V$. parahaemolyticus detected in those stages (Table 2, Figs. 1 \& 2). It appeared that the probability of detection of pathogenic $V$. parahaemolyticus was much lower than that of total $\mathrm{Vp}$, particularly in the egg and nauplii stages, and to a lesser extent during the PL to PD stages. The correlation between total $\mathrm{Vp}$ and the pathogenic $V$. parahaemolyticus was observed from the protozoea to the PE3 stages, indicating the association between the concentrations of the total $\mathrm{Vp}$ and $t d h^{+} \mathrm{Vp}$ and $t r h^{+} \mathrm{Vp}$ in those stages. However, according to the Pearson's correlation coefficient, a direct correlation between total $\mathrm{Vp}$ and pathogenic Vp was detected only during the PE3 stage $(r=0.76)$ (Fig. 5).

The uncertainty of probability was scrutinized by Monte Carlo simulation; it was found that the probability of detection of pathogenic $V$. parahaemolyticus was significantly lower than that of total $\mathrm{Vp}$ in the egg, nauplii, PA, PC, and PD stages $(\mathrm{p}<0.05)$. Surprisingly, the probability of detection of pathogenic $V$. parahaemolyticus was statistically different from that of total Vp in the PE1 stage $(\mathrm{p}<0.05)$ since the uncertainty of probability of detection of total $\mathrm{Vp}$ was not detected from the simulation. A sensitivity analysis of probability was performed using the correlation between probability and its input variables such as water, sediment, larva, etc. The correlations between

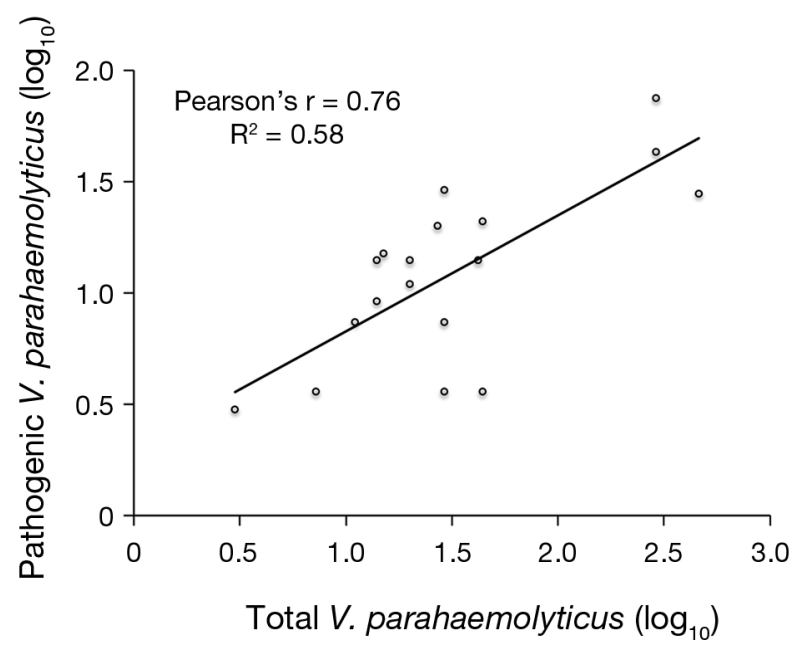

Fig. 5. Correlation between total Vibrio parahaemolyticus (total Vp) and pathogenic V. parahaemolyticus (patho Vp). Each dot represents 1 sample $(\mathrm{n}=18)$; the straight line represents the best-fit line obtained by linear regression analysis 
the probability of pathogenic $V$. parahaemolyticus and the prevalence of pathogenic $V$. parahaemolyticus in the water and sediment were between 0.60 and 0.67, particularly in the PA, PC, and PD stages (data not shown). The correlations between probability of pathogenic $V$. parahaemolyticus and prevalence of pathogenic $V$. parahaemolyticus in PL or shrimp and feed were around 0.37-0.40. Thus, we concluded that the important factors involved in the probability of detection of pathogenic $V$. parahaemolyticus in shrimp culture are water and sediment rather than PL or shrimp and feed.

\section{DISCUSSION}

In this study, the prevalence and concentration of the total and pathogenic Vibrio parahaemolyticus were determined during a full shrimp culture cycle using the MPN technique. In the hatchery cycle, it was clearly demonstrated that broodstock, eggs, and nauplii were the predominant sources of total $\mathrm{Vp}$ (Table 2, Fig. 1). Nonetheless, this bacterium may be a part of shrimp normal flora, and vertical transmission of organisms from broodstock to nauplii is common (Pangastuti et al. 2010). We found that around $83 \%$ of fresh feed that was provided to the broodstock for around 1 mo before spawning were positive for total $\mathrm{Vp}$ (Table 2). Thus, a repeat contamination of this organism at the broodstock stage might come from the fresh feed and could be transferred to the nauplii during larval rearing.

In this study, the prevalence and concentration of $V$. parahaemolyticus in the protozoea, mysis and PL stages was low because the hatching nauplii were filtered and transferred to a new tank (rearing tank) with new water supplied continuously. In addition, all the feed supplied in those stages were live or boiled artemia, spirulina, and formulated feed that for the most part had no $V$. parahaemolyticus $\left(-0.53 \log _{10}\right.$ MPN g ${ }^{-1}$ or ml-1) (Table 2, Fig. 3). Only one sample of artemia was positive for $V$. parahaemolyticus.

In the pond cycle during the PA-PC stages, management of the pond was quite good as less than $50 \%$ of the samples were positive for total $\mathrm{Vp}$ (Table 2). The sediment had more positives than the water samples (Fig. 2). However, during the PE1PE3 stages, the prevalence and concentration of $V$. parahaemolyticus increased continuously. This may be due to repeat contamination of this bacterium from the PL released into the pond (PD stage) because 3 out of 24 samples of PL (12.5\%) were positive for total Vp (data not shown). Together with the
$V$. parahaemolyticus in the pond and the organic matter from the formulated feed supplied for shrimp in this culture cycle, these could be the important factors responsible for the increase of this bacterium during the PE1-PE3 stages. Most of the formulated feed was composed of ground and sieved fish, soybean, starch, fat, and oils.

In this study, the use of the LAMP technique for detection of pathogenic $V$. parahaemolyticus in the supernatant of the positive MPN tubes produced greater numbers than the colony PCR, as it detected an additional $t r h^{+} \mathrm{Vp}$ sample in the broodstock, detected $t d h^{+} \mathrm{Vp}$ and $t r h^{+} \mathrm{Vp}$ in samples obtained during the PE1 to PE3 stages, and showed higher numbers of positive samples for $t d h^{+} \mathrm{Vp}$ and detected $t r h^{+} \mathrm{Vp}$ in samples of fresh feed (Table 2). It is unlikely that this increase in detection is due to nonspecific detection because all of the positive tubes were confirmed for the presence of $V$. parahaemolyticus. In this work, in comparison to PCR, the sensitivity of LAMP for detection of both $t d h$ and trh was $100 \%$ whereas the specificity for detection of $t d h$ and trh were 92.1 and 97.9\%, respectively (Table 3). These estimates are, however, dependent upon the assumption that PCR is a gold standard test, and the estimates may, therefore, be biased. The LAMP technique can also discriminate between the trh subgroups (Yamazaki et al. 2010). In this study, all 4 samples obtained from the PE3 samples were positive for trh2 whereas only 1 and 2 samples obtained from fresh feed were positive for trh1 and trh2, respectively (Table 2).

In our study, the probability of detection of total $\mathrm{Vp}$ and pathogenic $V$. parahaemolyticus were evaluated to assess their correlations. The probability of detection of pathogenic $V$. parahaemolyticus was lower than that of total Vp in the early period of the hatchery cycle (egg and nauplii stages) and the pond cycle (PA, PC, and PD stages). In addition, a direct correlation between total $\mathrm{Vp}$ and pathogenic $V$. parahaemolyticus was observed only during the PE3 stage (Fig. 5). Although these stages constituted less than half of the total stages in the shrimp culture, this indicated that total Vp might not be a good candidate to represent the pathogenic $V$. parahaemolyticus in the shrimp culture.

In this work, the factors involved in probability evaluation were the detection and enumeration of $V$. parahaemolyticus from various sources in each stage of shrimp culture. Therefore, identification of the sources contaminated with pathogenic $V$. parahaemolyticus either from the environment or from shrimp was of interest. We found that the correlation 
between the probability of detection of pathogenic $V$. parahaemolyticus from the environment (water and sediment) was higher than that of detection from shrimp ( $\mathrm{r}=0.60-0.67)$. This indicated that the important sources of pathogenic $V$. parahaemolyticus were water and sediment.

After 4.5 mo of shrimp culture (PE3), the average concentrations of both the total $\mathrm{Vp}$ and the pathogenic $\mathrm{Vp}$ were higher than those detected after 1.5 and 3 mo culture (PE1 and PE2, respectively) (Fig. 2). During the PE3 stage, the maximum concentration of the total Vp was $4.0 \pm 0.48 \log _{10} \mathrm{MPN} \mathrm{g}^{-1} \mathrm{or} \mathrm{ml}^{-1}$, and it was $3.1 \pm 0$ and $3.1 \pm 0.48 \log _{10} \mathrm{MPN} \mathrm{g}^{-1}$ or ml $^{-1}$ in the PE1 and PE2, respectively. For the pathogenic $\mathrm{Vp}$, the maximum concentration detected in PE3 was $1.2 \pm 0 \log _{10} \mathrm{MPN} \mathrm{g}^{-1}$ or $\mathrm{ml}^{-1}$, and it was $0.5 \pm 0$ and $0.8 \pm 0 \log _{10} \mathrm{MPN} \mathrm{g}^{-1}$ or $\mathrm{ml}^{-1}$ in the PE1 and PE2, respectively. A correlation between the total $\mathrm{Vp}$ and pathogenic Vp was detected only in the PE3 stage (Fig. 5). Thus, the correlation in terms of the concentration between the total and pathogenic $V$. parahaemolyticus might be more apparent, particularly when the concentrations of the total and pathogenic $V$. parahaemolyticus reached a higher level of contamination.

Recently, $V$. parahaemolyticus has been demonstrated to be one of etiologic agents of acute hepatopancreatic necrosis disease (AHPND) (Tran et al. 2013). Outbreaks in shrimp culture occur within the first $30 \mathrm{~d}$ after releasing PL into the shrimp pond. Mass mortality can exceed $70 \%$ of the total shrimp and cause mass economic losses for the shrimp industry. However, V. parahaemolyticus that causes AHPND, lacks both the $t d h$ and $t r h$ virulence genes, and it has not been reported to be associated with any human disease. The scenario used in this study will be useful for illustrating the source of this bacterium as well as of other shrimp pathogen contamination during the shrimp culture stages.

In conclusion, this study has illustrated that in shrimp culture, the prevalence and concentration of $V$. parahaemolyticus was high at the beginning of the hatchery cycle, and the presence of this organism decreased in the subsequent larval and PL stages. In contrast, they were low during the beginning of the pond cycle, yet dramatically increased in the later stages of culture. Broodstock and fresh feed were important sources of $V$. parahaemolyticus; however, water and sediment were involved in the probability of detection of pathogenic $V$. parahaemolyticus. A direct correlation between total and pathogenic $V$. parahaemolyticus was observed in the harvest stage. This study will be useful as a guideline to establish a level for the presence of pathogenic strains which is considered safe and, thus, decrease the risk for people who consume shrimp.

Acknowledgements. This work was supported by funds from the National Science and Technology Development Agency (NSTDA), Thailand (TG-CPMO 01-54-001) and the Thailand Research Fund through the Royal Golden Jubilee PhD Program (grant no. PHD/0066/2550). Thanks to Dr. Brian Hodgson for assistance with the manuscript.

\section{LITERATURE CITED}

Cassin MH, Lammerding AM, Todd ECD, Ross W, McColl RS (1998) Quantitative risk assessment for Escherichia coli O157:H7 in ground beef hamburgers. Int J Food Microbiol 41:21-44

Crépet A, Albert I, Dervin C, Carlin F (2007) Estimation of microbial contamination of food from prevalence and concentration data: application to Listeria monocytogenes in fresh vegetables. Appl Environ Microbiol 73:250-258

Deepanjali A, Sanath Kumar H, Karunasagar I, Karunasagar I (2005) Seasonal variation in abundance of total and pathogenic Vibrio parahaemolyticus bacteria in oysters along the Southwest Coast of India. Appl Environ Microbiol 71:3575-3580

FDA (2011) FDA/CFSAN NSSP guide for the control of molluscan shellfish. www.fda.gov/Food/GuidanceRegulation/ FederalStateFoodPrograms/ucm2006754.htm

Geng S, Campbell RN, Carter M, Hills FJ (1983) Quality control of plant pathogens. Plant Dis 67:236-242

Gutierrez West CK, Klein SL, Lovell CR (2013) High frequency of virulence factor genes $t d h, t r h$, and $t h$ in Vibrio parahaemolyticus strains isolated from a pristine estuary. Appl Environ Microbiol 79:2247-2252

> Honda S, Goto I, Minematsu I, Ikeda N and others (1987) Gastroenteritis due to Kanagawa negative Vibrio parahaemolyticus. Lancet 329:331-332

> Lau YL, Meganathan P, Sonaimuthu P, Thiruvengadam G, Nissapatorn V, Chen Y (2010) Specific, sensitive, and rapid diagnosis of active toxoplasmosis by a loopmediated isothermal amplification method using blood samples from patients. J Clin Microbiol 48:3698-3702

Liu M, Chen S (2013) Draft genome sequence of Vibrio parahaemolyticus V110, isolated from shrimp in Hong Kong. Genome Announc 1:e00300-e00313

Murray N, Macdiarmid S, Wooldridge M, Gummon B, Morley RS, Weber SE, Giovannini A, Wilson D (2004) Quantitative risk assessment. In: Murray N (ed) Handbook on import risk analysis for animals and animal products: quantitative risk assessment, Vol 2. Office International des Épizooties, Paris, p 35

Pangastuti A, Suwanto A, Lestari Y, Suhartono MT (2010) Bacterial communities associated with white shrimp (Litopenaeus vannamei) larvae at early developmental stages. Biodiversitas 11:65-68

Rahimi E, Ameri M, Doosti A, Gholampour AR (2010) Occurrence of toxigenic Vibrio parahaemolyticus strains in shrimp in Iran. Foodborne Pathog Dis 7:1107-1111

Sujeewa AKW, Norrakiah AS, Laina M (2009) Prevalence of toxic genes of Vibrio parahaemolyticus in shrimps (Penaeus monodon) and culture environment. Int Food Res J 16:89-95 
Takeda Y (1982) Thermostable direct hemolysin of Vibrio parahaemolyticus. Pharmacol Ther 19:123-146

Tran L, Nunan L, Redman RM, Mohney LL, Pantoja CR, Fitzsimmons K, Lightner DV (2013) Determination of the infectious nature of the agent of acute hepatopancreatic necrosis syndrome affecting penaeid shrimp. Dis Aquat Org 105:45-55

Wootipoom N, Bhoopong P, Pomwised R, Nishibuchi M, Ishibashi M, Vuddhakul V (2007) A decrease in proportion of infections by pandemic Vibrio parahaemolyticus in Hat Yai Hospital, southern Thailand. J Med Microbiol 56:1630-1638

Yamamoto A, Iwahori J, Vuddhakul V, Charernjiratragul W and others (2008) Quantitative modeling for risk assessment of Vibrio parahaemolyticus in bloody clams in southern Thailand. Int J Food Microbiol 124: 70-78

Yamazaki W, Ishibashi M, Kawahara R, Inoue K (2008) Development of a loop-mediated isothermal amplification assay for sensitive and rapid detection of Vibrio parahaemolyticus. BMC Microbiol 8:163

Yamazaki W, Kumeda Y, Misawa N, Nakaguchi Y, Nishibuchi M (2010) Development of a loop-mediated isothermal amplification assay for sensitive and rapid detection of the tdh and trh genes in Vibrio parahaemolyticus and related Vibrio species. Appl Environ Microbiol 76: 820-828

Yeung PMS, Boor KJ (2004) Epidemiology, pathogenesis, and prevention of foodborne Vibrio parahaemolyticus infections. Foodborne Pathog Dis 1:74-88

\section{Appendix}

For investigation of the probability of detection of Vibrio parahaemolyticus in samples, $P_{\mathrm{c}}$ represents the probability of the presence of a pathogen in a sample unit and $P_{\mathrm{s}}$ represents the prevalence of contaminated samples; thus, the probability of exposure to $V$. parahaemolyticus from a sample unit assay, $P_{\mathrm{e}}$ (Cassin et al. 1998), is:

$$
P_{\mathrm{e}}=P_{\mathrm{C}} \times P_{\mathrm{S}}
$$

The probability of detecting $V$. parahaemolyticus in a sample depends on the concentration of this bacterium $(C)$ in each sample type of each culture stage and the quantity of the corresponding sample tested $\left(N_{i} \mathrm{~g}^{-1}\right.$ or ml $\mathrm{m}^{-1}$ of sample). The concentration variable was described by lognormal distribution $(\mu, \sigma)$. This probability is assumed to follow a Poisson distribution (Crépet et al. 2007):

$$
P_{\mathrm{c}}=1-\mathrm{e}^{(-\mathrm{NC})}
$$

The prevalence $\left(P_{\mathrm{s}}\right)$ of contaminated sample depends on numbers of both contaminated samples $(s)$ and sample size $(k)$. In order to avoid problems that arise from $0 \%$ prevalence, the expected value from the beta distribution was used as the prevalence since the domain of the beta distribution encompasses all possible values of prevalence. The 2 parameters of the beta distribution are $\alpha(\alpha=s+1)$ and $\beta$ $(\beta=k-s+1)$ (Murray et al. 2004):

$$
P_{\mathrm{s}}=\operatorname{Beta}(s+1, k-s+1)
$$

From Eqs. (1) \& (2), both $P_{\mathrm{c}}$ and $P_{\mathrm{s}}$ were the basis of contamination of $V$. parahaemolyticus in a single sampling. If repeated samplings (with an equal number of sample units in all sets of sampling) have been drawn $k$ times, the probability of having $V$. parahaemolyticus at least 1 time from total $k$ times was calculated as the following (Geng et al. 1983):

$$
P_{+}=1-\left(1-P_{\mathrm{e}}\right)^{k}
$$

During the shrimp cultivation, $V$. parahaemolyticus could be derived from shrimp itself or from some other possible source in the environment, e.g. sediment, feed, water, etc. It would be interesting to determine the probability of detecting $V$. parahaemolyticus from at least 1 source in each stage of cultivation. With the same analogy of Eq. (4), each sampling equation time was regarded as each source of this bacterium. In this case, $i$ would represent the number of possible sources of $V$. parahaemolyticus and $P_{\mathrm{e}_{i}}$ would represent $P_{\mathrm{e}}$ of each possible source of $V$. parahaemolyticus. Therefore, the probability of detecting $V$. parahaemolyticus in at least 1 source from total $k$ sources of this bacterium in each stage of shrimp cultivation was slightly redefined as the following:

$$
P_{+}=1-\prod_{i=1}^{k}\left(1-P_{\mathrm{e}_{i}}\right)^{k_{i}}
$$

In this study, for a sample containing no V. parahaemolyticus, $0.29 \mathrm{MPN} \mathrm{g}^{-1}$ (or ml$^{-1}$ ) will be used for the calculation (FDA 2011). In the case where all samples are positive for $V$. parahaemolyticus, the average concentration of this bacterium was increased by 1 significant number. For example, if the upper limit of the MPN estimation was $1100 \mathrm{MPN} \mathrm{g}^{-1}\left(\mathrm{or} \mathrm{ml}^{-1}\right)$, then the average concentration of V. parahaemolyticus was $1200 \mathrm{MPN} \mathrm{g}^{-1}$ (or $\mathrm{ml}^{-1}$ ) (FDA 2011).

The uncertainty of probability was described by the Monte Carlo simulation. The simulation was aimed at resampling all possible values of input variables such as concentration and prevalence by using the @Risk Decision tool version 4.5 (Palisade Corp.). The resamplings were repeated up to 10000 iterations.

Correlation between total $\mathrm{Vp}$ and $t d h^{+} \mathrm{Vp}$ and $t r h^{+} \mathrm{Vp}$ was determined by Pearson's product-moment correlation. 